\title{
The Representations and Continuity of the Metric Projections on Two Classes of Half-Spaces in Banach Spaces
}

\author{
Zihou Zhang and Chunyan Liu \\ College of Fundamental Studies, Shanghai University of Engineering Science, Shanghai 201620, China \\ Correspondence should be addressed to Zihou Zhang; zhzmath@gmail.com
}

Received 20 October 2013; Accepted 31 December 2013; Published 17 February 2014

Academic Editor: Khalil Ezzinbi

Copyright (C) 2014 Z. Zhang and C. Liu. This is an open access article distributed under the Creative Commons Attribution License, which permits unrestricted use, distribution, and reproduction in any medium, provided the original work is properly cited.

\begin{abstract}
We show a necessary and sufficient condition for the existence of metric projection on a class of half-space $K_{x_{0}^{*}, c}=\left\{x \in X: x^{*}(x) \leq\right.$ $c\}$ in Banach space. Two representations of metric projections $P_{K_{x_{0}^{*}, c}}$ and $P_{K_{x_{0}, c}}$ are given, respectively, where $K_{x_{0}, c}$ stands for dual half-space of $K_{x_{0}^{*}, c}$ in dual space $X^{*}$. By these representations, a series of continuity results of the metric projections $P_{K_{x_{0}^{*}, c}}$ and $P_{K_{x_{0}, c}}$ are given. We also provide the characterization that a metric projection is a linear bounded operator.
\end{abstract}

\section{Introduction}

The metric projection in Banach space is an enduring question for study or discussion. It has been used in many areas of mathematics such as the theories of optimization and approximation, fixed point theory, nonlinear programming, and variational inequalities. On continuity of the metric projection, many mathematicians, for example, Nevesenko [1], Oshman [2], Wang [3], Fang and Wang [4], and Zhang and Shi [5] have done profound research. In practical application, giving the representations of metric projection is very necessary. Generally speaking, this is very difficult. In recent years, Wang and $\mathrm{Yu}$ [6] gave a representation of single-valued metric projection on a class of hyperplanes $H_{x_{0}^{*}, c}=\{x \in$ $X: x_{0}^{*}(x)=c$ \} in reflexive, smooth, and strictly convex Banach space $X$. Song and Cao [7] gave a representation of metric projection on a class of half-space $K_{x_{0}^{*}, c}$ in the reflexive, smooth, and strictly convex Banach space $X$. Wang [8] and $\mathrm{Ni}[9]$ extended the result of Wang and Yu to general Banach space, respectively. Wang [8] also discussed continuity of the metric projection on the hyperplane $H_{x_{0}^{*}, c}$ in Banach space.

In this paper, let $X$ be a Banach space and let $X^{*}$ be the dual of $X$. Let $S(X)$ and $B(X)$ be the unit sphere and unit ball of $X$, respectively. Let $x_{0}^{*} \in X^{*} \backslash\{\theta\}$, let $c \in \mathbb{R}$, let $K_{x_{0}^{*}, c}=$ $\left\{x \in X: x_{0}^{*}(x) \leq c\right\}$, let $D^{-1}\left(x_{0}^{*}\right)=\left\{x \in X: x_{0}^{*}(x)=\right.$ $\left.\left\|x_{0}^{*}\right\|\|x\|=\left\|x_{0}^{*}\right\|^{2}=\|x\|^{2}\right\}$, let $x_{0} \in X \backslash\{\theta\}$, let $K_{x_{0}, c}=\left\{x^{*} \in\right.$
$\left.X^{*}: x^{*}\left(x_{0}\right) \leq c\right\}$, and let $D\left(x_{0}\right)=\left\{x^{*} \in X^{*}: x^{*}\left(x_{0}\right)=\right.$ $\left.\left\|x^{*}\right\|\left\|x_{0}\right\|=\left\|x^{*}\right\|^{2}=\left\|x_{0}\right\|^{2}\right\}$. It is easily proved that $D(\alpha x)=$ $\alpha D(x), D^{-1}\left(\alpha x^{*}\right)=\alpha D^{-1}\left(x^{*}\right)$, and for all $\alpha \in \mathbb{R}$. For $M \subset X$, the metric projection $P_{M}: X \rightarrow 2^{M}$ is defined by $P_{M}(x)=$ $\{y \in M:\|x-y\|=d(x, M)\}$, where $d(x, M)=\inf \{\|x-y\|:$ $y \in M$. Obviously, $P_{M}$ is a set-valued mapping. If $P_{M}(x) \neq \emptyset$ for each $x \in X$, then $M$ is said to be proximinal. It is well known that $P_{M}$ is single-valued when $X$ is strictly convex and $M$ is proximal.

Cabrera and Sadarangani [10] introduced the geometrical properties of Banach spaces as follows.

A Banach space $X$ is called nearly strictly convex (resp., weakly nearly strictly convex) whenever, for any $x^{*} \in S\left(X^{*}\right)$, the set $D^{-1}\left(x^{*}\right)$ is compact (resp., weakly compact). A Banach space $X$ is called nearly smooth (resp., weakly nearly smooth) whenever, for any $x \in S(X)$, the set $D(x)$ is compact (resp., weakly compact).

The metric projection $P_{M}$ is said to be norm-norm (resp., norm-weakly) upper semicontinuous if, for all $x$ in $X$ and for all norm (resp., weakly) open set $W \supset P_{M}(x)$, there exists a norm neighborhood $U$ of $x$ such that $P_{M}(U) \subset W$.

In this paper, firstly, we established a necessary and sufficient condition for the existence of metric projection on a class of half-space $K_{x_{0}^{*}, c}$ in Banach space. Secondly, we give two representations of the metric projections $P_{K_{x_{0}^{*}, c}}$ and 
$P_{K_{x_{0}, c}}$ by using a different method from the literatures [5-9]. Thirdly, by these representations, we prove that if $X$ is weakly nearly strictly convex (resp., weakly nearly smooth), then metric projection $P_{K_{x_{0}^{*}, c}^{*}}\left(\right.$ resp., $\left.P_{K_{x_{0}, c}}\right)$ is norm-weakly upper semicontinuous. Finally, the characterization of the metric projection $P_{M}$ from $X$ to a subspace $M$ to be a linear bounded operator is given. We extend the corresponding results in [59].

\section{The Representations of the Metric Projection on Two Classes of Half-Spaces in Banach Spaces}

Lemma 1. Let $X$ be a Banach space and let $x_{0}^{*} \in X^{*} \backslash\{\theta\}$; then $d\left(x, K_{x_{0}^{*}, c}\right)=\left|x_{0}^{*}(x)-c\right| /\left\|x_{0}^{*}\right\|$ for all $x \in X \backslash K_{x_{0}^{*}, c}$.

Proof. Firstly, suppose that $\left\|x_{0}^{*}\right\|=1$. Let $x \in X / K_{x_{0}^{*}, c}$. For any $y \in K_{x_{0}^{*}, c}$, since

$$
x_{0}^{*}(x-y) \geq x_{0}^{*}(x)-c>0,
$$

we deduce that

$$
d\left(x, K_{x_{0}^{*}, c}\right) \geq\left|x_{0}^{*}(x)-c\right| .
$$

On the other hand, for any $\varepsilon>0(\varepsilon<1 / 4)$, there exists $u_{\varepsilon}$ in $S(X)$ such that $1-\varepsilon<x_{0}^{*}\left(u_{\varepsilon}\right) \leq 1$. Set $y_{\varepsilon}=x-(1+$ $2 \varepsilon)\left(x_{0}^{*}(x)-c\right) u_{\varepsilon}$. Then

$$
\begin{aligned}
x_{0}^{*}\left(y_{\varepsilon}\right) & =x_{0}^{*}(x)-(1+2 \varepsilon)\left(x_{0}^{*}(x)-c\right) x_{0}^{*}\left(u_{\varepsilon}\right) \\
& <x_{0}^{*}(x)-(1+2 \varepsilon)\left(x_{0}^{*}(x)-c\right)(1-\varepsilon) \\
& =x_{0}^{*}(x)-\left(1+\varepsilon-2 \varepsilon^{2}\right)\left(x_{0}^{*}(x)-c\right) \\
& \leq x_{0}^{*}(x)-\left(x_{0}^{*}(x)-c\right)=c .
\end{aligned}
$$

Consequently, $y_{\varepsilon} \in K_{x_{0}^{*}, c}$ and

$$
\left\|x-y_{\varepsilon}\right\|=(1+2 \varepsilon)\left|x_{0}^{*}(x)-c\right| \text {. }
$$

It follows that

$$
d\left(x, K_{x_{0}^{*}, c}\right) \leq(1+2 \varepsilon)\left|x_{0}^{*}(x)-c\right| .
$$

By arbitrariness of $\varepsilon$, we deduce that

$$
d\left(x, K_{x_{0}^{*}, c}\right) \leq\left|x_{0}^{*}(x)-c\right| .
$$

This means that

$$
d\left(x, K_{x_{0}^{*}, c}\right)=\left|x_{0}^{*}(x)-c\right| .
$$

Secondly, for $x^{*} \in X^{*} \backslash \theta$ and $\left\|x^{*}\right\| \neq 1$, since

$$
K_{x_{0}^{*}, c}=\left\{x \in X: x_{0}^{*}(x) \leq c\right\}=\left\{x \in X: \frac{x_{0}^{*}}{\left\|x_{0}^{*}\right\|}(x) \leq \frac{c}{\left\|x_{0}^{*}\right\|}\right\},
$$

from (7), we may obtain that

$$
d\left(x, K_{x_{0}^{*}, c}\right)=\frac{\left|x_{0}^{*}(x)-c\right|}{\left\|x_{0}^{*}\right\|}, \quad \forall x \in X \backslash K_{x_{0}^{*}, c} .
$$

Remark 2. For given $x_{0}^{*} \in X^{*} \backslash\{\theta\}$ and $c \in \mathbb{R}$, by Lemma 1 , we have that

$$
d\left(x, K_{x_{0}^{*}, c}\right)=d\left(x, H_{x_{0}^{*}, c}\right),
$$

for any $x \in X \backslash K_{x_{0}^{*}, c}$.

Theorem 3. Let $X$ be a Banach space, let $x_{0}^{*} \in X^{*} \backslash \theta$, and let $c \in \mathbb{R}$; then $P_{K_{x_{0}^{*}, c}}(x) \neq \emptyset$ for any $x \in X$ if and only if $D^{-1}\left(x_{0}^{*}\right) \neq \emptyset$.

Proof. On necessity: take $x \in X \backslash K_{x_{0}^{*}, c}$; then there exists a $y \in P_{K_{x_{0}^{*}, c}^{*}}(x)$. Set $u=\left(\left\|x_{0}^{*}\right\|^{2} /\left(x_{0}^{*}(x)-c\right)\right)(x-y)$; by Lemma 1 , we have that

$$
\begin{aligned}
\|u\| & =\frac{\left\|x_{0}^{*}\right\|^{2}}{\left|x_{0}^{*}(x)-c\right|}\|x-y\| \\
& =\frac{\left\|x_{0}^{*}\right\|^{2}}{\left|x_{0}^{*}(x)-c\right|} \frac{\left|x_{0}^{*}(x)-c\right|}{\left\|x_{0}^{*}\right\|}=\left\|x_{0}^{*}\right\| .
\end{aligned}
$$

Hence, $x_{0}^{*}(u) \leqslant\left\|x_{0}^{*}\right\|\|u\|=\left\|x_{0}^{*}\right\|^{2}$.

On the other hand,

$$
\begin{aligned}
x_{0}^{*}(u) & =\frac{\left\|x_{0}^{*}\right\|^{2}}{x_{0}^{*}(x)-c}\left(x_{0}^{*}(x)-x_{0}^{*}(y)\right) \\
& \geqslant \frac{\left\|x_{0}^{*}\right\|^{2}}{x_{0}^{*}(x)-c}\left(x_{0}^{*}(x)-c\right)=\left\|x_{0}^{*}\right\|^{2} .
\end{aligned}
$$

This shows that $x_{0}^{*}(u)=\left\|x_{0}^{*}\right\|^{2}=\|u\|^{2}$, that is, $u \in D^{-1}\left(x_{0}^{*}\right)$ and $D^{-1}\left(x_{0}^{*}\right) \neq \emptyset$.

On sufficiency: take $x \in S(X)$ such that $x_{0}^{*}(x)=$ $\left\|x_{0}^{*}\right\|\|x\|=\left\|x_{0}^{*}\right\|^{2}=\|x\|^{2}$. We discuss that in two cases.

Case 1. If $x \in K_{x_{0}^{*}, c}$, then $x \in P_{K_{x_{0}^{*}, c}}(x)$.

Case 2. If $x \notin K_{x_{0}^{*}, c}$, since

$$
x_{0}^{*}\left(x-\frac{x_{0}^{*}(x)-c}{\left\|x_{0}^{*}\right\|^{2}} x_{0}\right)=x_{0}^{*}(x)-\left(x_{0}^{*}(x)-c\right)=c ;
$$

then we have that $x-\left(\left(x_{0}^{*}(x)-c\right) /\left\|x_{0}^{*}\right\|^{2}\right) x_{0} \in K_{x_{0}^{*}, c}$. By Lemma 1,

$$
\left\|x-\left(x-\frac{x_{0}^{*}(x)-c}{\left\|x_{0}^{*}\right\|^{2}} x_{0}\right)\right\|=\frac{x_{0}^{*}(x)-c}{\left\|x_{0}^{*}\right\|}=d\left(x, K_{x_{0}^{*}}\right) .
$$

It follows that $x-\left(\left(x_{0}^{*}(x)-c\right) /\left\|x_{0}^{*}\right\|^{2}\right) x_{0} \in P_{K_{x_{0}^{*}, c}^{*}}(x)$.

Theorem 4. Let $X$ be a Banach space, let $x_{0}^{*} \in X^{*} \backslash\{\theta\}$, let $x_{0}^{*}$ attain its norm on $S(X)$, and let $c \in \mathbb{R}$. Then

$$
P_{K_{x_{0}^{*}, c}^{*}}(x)=x-\max \left\{0, \frac{x_{0}^{*}(x)-c}{\left\|x_{0}^{*}\right\|^{2}}\right\} D^{-1}\left(x_{0}^{*}\right) .
$$


Proof. Take $x \in X$. We discuss that in two cases.

Case 1. If $x \in K_{x_{0}^{*}, c}$, then $P_{K_{x_{0}^{*}, c}}(x)=\{x\}$.

Case 2. If $x \notin K_{x_{0}^{*}, c}$, we arbitrarily take $x_{0} \in D^{-1}\left(x_{0}^{*}\right)$. Let $y=$ $x-\left(\left(x_{0}^{*}(x)-c\right) /\left\|x_{0}^{*}\right\|^{2}\right) x_{0}$. Similar to the proof of Theorem 3 , we may obtain that $y \in P_{K_{x_{0}^{*}, c}}(x)$. Therefore,

$$
x-\frac{x_{0}^{*}(x)-c}{\left\|x_{0}^{*}\right\|^{2}} D^{-1}\left(x_{0}^{*}\right) \subset P_{K_{x_{0}, c}^{*}}(x) .
$$

On the other hand, we arbitrarily take $y \in P_{K_{x_{0}^{*}, c}}(x)$. Let $u=\left(\left\|x_{0}^{*}\right\|^{2} /\left(x_{0}^{*}(x)-c\right)\right)(x-y)$; similar to the proof of Theorem 3, we may obtain that $u \in D^{-1}\left(x_{0}^{*}\right)$. Therefore,

$$
y=x-\frac{x_{0}^{*}(x)-c}{\left\|x_{0}^{*}\right\|^{2}} u \in x-\frac{x_{0}^{*}(x)-c}{\left\|x_{0}^{*}\right\|^{2}} D^{-1}\left(x_{0}^{*}\right),
$$

that is,

$$
P_{K_{x_{0}, c}^{*}}(x) \subset x-\frac{x_{0}^{*}(x)-c}{\left\|x_{0}^{*}\right\|^{2}} D^{-1}\left(x_{0}^{*}\right) .
$$

By Case 1 and Case 2, we have

$$
P_{K_{x_{0}^{*}, c}}(x)=x-\max \left\{0, \frac{x_{0}^{*}(x)-c}{\left\|x_{0}^{*}\right\|^{2}}\right\} D^{-1}\left(x_{0}^{*}\right),
$$

for any $x \in X$.

By the similar proof to that in Lemma 1, we can obtain the following result.

Lemma 5. Let $X$ be a Banach space, let $x_{0} \in X \backslash\{\theta\}$, and let $c \in \mathbb{R}$. Then

$$
d\left(x^{*}, K_{x_{0}, c}\right)=\frac{\left|x^{*}\left(x_{0}\right)-c\right|}{\left\|x_{0}\right\|},
$$

for any $x^{*} \in X^{*} \backslash K_{x_{0}, c}$.

By a similar proof to that in Theorem 4, we can also prove the following result according to Lemma 5.

Theorem 6. Let $X$ be a Banach space, let $x_{0} \in X \backslash\{\theta\}$, and let $c \in \mathbb{R}$. Then

$$
P_{K_{x_{0}, c}}\left(x^{*}\right)=x^{*}-\max \left\{0, \frac{x^{*}\left(x_{0}\right)-c}{\left\|x_{0}\right\|^{2}}\right\} D\left(x_{0}\right),
$$

for any $x^{*} \in X^{*}$.

\section{Continuity of the Metric Projection on the Two Classes of Half-Spaces in Banach Spaces}

Theorem 7. Let $x_{0}^{*} \in X \backslash\{\theta\}$, let $x_{0}^{*}$ attain its norm on $S(X)$, and let $c \in \mathbb{R}$. If $X$ is weakly nearly strictly convex, then the metric projection $P_{K_{x_{0}^{*}, c}^{*}}$ is norm-weakly upper semicontinuous.
Proof. Let $x, x_{n} \in X$, and let $x_{n} \rightarrow x$ as $n \rightarrow \infty$. Our proof will be divided into two cases.

Case 1. Suppose that $\left\{x_{n}\right\} \subset K_{x_{0}^{*}, c}$. Since $K_{x_{0}^{*}, c}$ is a closed set, $x \in K_{x_{0}^{*}, c}$. Clearly, $P_{K_{x_{0}^{*}, c}}\left(x_{n}\right)=x_{n} \rightarrow x=P_{K_{x_{0}^{*}, c}}(x)$.

Case 2. Suppose that $\left\{x_{n}\right\} \not \subset K_{x_{0}^{*}, c}$.

If there are an infinite number of $n$ for which $x_{n} \in K_{x_{0}^{*}, c}$, then we can choose a subsequence $\left\{x_{n_{k}}\right\} \subset\left\{x_{n}\right\}$ with $\left\{x_{n_{k}}\right\} \subset$ $K_{x_{0}^{*}, c}$. Therefore, $P_{K_{x_{0}^{*}, c}}\left(x_{n_{k}}\right)=x_{n_{k}} \rightarrow x=P_{K_{x_{0}^{*}, c}}(x)$ as $k \rightarrow$ $\infty$.

If there are an infinite number of $n$ for which $x_{n} \notin K_{x_{0}^{*}, c}$, without loss of generality, we may assume that $\left\{x_{n}\right\} \subset X \backslash$ $K_{x_{0}^{*}, c}$. Taking $y_{n} \in P_{K_{x_{0}^{*}, c}}\left(x_{n}\right)$, by Theorem 4 , we have

$$
P_{K_{x_{0}^{*}, c}}\left(x_{n}\right)=x_{n}-\frac{x_{0}^{*}\left(x_{n}\right)-c}{\left\|x_{0}\right\|^{2}} D^{-1}\left(x_{0}^{*}\right) \text {. }
$$

We assume that $y_{n}=x_{n}-\left(\left(x_{0}^{*}\left(x_{n}\right)-c\right) /\left\|x_{0}^{*}\right\|^{2}\right) z_{n}$, where $z_{n} \in D^{-1}\left(x_{0}^{*}\right)$. Since $X$ is weakly nearly strictly convex, we know that $\left\{z_{n}\right\}$ has a weakly convergent subsequence $\left\{z_{n_{k}}\right\}$ with $z_{n_{k}} \stackrel{w}{\rightarrow} z$ as $k \rightarrow \infty$. Consequently,

$$
y_{n_{k}}=x_{n_{k}}-\frac{x_{0}^{*}\left(x_{n_{k}}\right)-c}{\left\|x_{0}^{*}\right\|} z_{n_{k}} \stackrel{w}{\longrightarrow} x-\frac{x_{0}^{*}(x)-c}{\left\|x_{0}^{*}\right\|} z .
$$

Noting $x_{0}^{*}(z)=\lim _{k} x_{0}^{*}\left(z_{n_{k}}\right)=\lim _{k}\left\|x_{0}^{*}\right\|^{2}=\left\|z_{n_{k}}\right\|^{2}$ and $\|z\| \leqslant \lim _{\bar{k}}\left\|z_{n_{k}}\right\|$, we know that $x_{0}^{*}(z) \geqslant\left\|x_{0}^{*}\right\| \cdot\|z\|$. Therefore,

$$
x_{0}^{*}(z)=\left\|x_{0}^{*}\right\| \cdot\|z\|=\left\|x_{0}^{*}\right\|^{2}=\|z\|^{2},
$$

where $z \in D^{-1}\left(x_{0}^{*}\right)$. This shows that $y_{n_{k}} \stackrel{w}{\rightarrow} x-\left(\left(x_{0}^{*}(x)-\right.\right.$ c) $\left./\left\|x_{0}^{*}\right\|\right) z \in P_{K_{x_{0}^{*}, c}}(x)$.

Now, we will show that $P_{K_{x_{0}^{*}, c}}$ is norm-weakly upper semicontinuous at $x$. Otherwise, there exist a weakly open set $W_{0} \supset P_{K_{x_{0}^{*}, c}}(x)$ and a sequence $\left\{x_{m}\right\}$ with $x_{m} \rightarrow x$ as $m \rightarrow \infty$, but $P_{K_{x_{0}^{*}, c}}\left(x_{m}\right) \not \subset W_{0}$ for all $m$. Taking $y_{m} \in$ $P_{K_{x_{0}^{*}, c}}\left(x_{m}\right) \backslash W_{0}, m=1,2, \ldots$, similar to previous arguments, we can observe the fact that there exists a subsequence $\left\{y_{m_{k}}\right\}$ of $\left\{y_{m}\right\}$ such that $y_{m_{k}} \stackrel{w}{\rightarrow} y$ as $k \rightarrow \infty$ and $y \in P_{K_{x_{0}^{*}, c}}(x)$. This means that there exists $y_{m_{k}} \in W_{0}$ for some $k$ large enough, which is a contradiction.

Similar to the proof of Theorem 8, we may prove the following theorem.

Theorem 8. Let $X$ be a Banach space.

(1) Let $x_{0}^{*} \in X^{*} \backslash\{\theta\}$, let $x_{0}^{*}$ attain its norm on $S(X)$, and let $c \in \mathbb{R}$. If $X$ is nearly strictly convex, then the metric projection $P_{K_{x_{0}^{*}, c}}$ is norm-norm upper semicontinuous.

(2) Let $x_{0} \in X \backslash\{\theta\}$ and let $c \in \mathbb{R}$. If $X$ is weakly nearly smooth, then the metric projection $P_{K_{x_{0}, c}}$ is normweakly upper semicontinuous. 
(3) Let $x_{0} \in X \backslash\{\theta\}$ and let $c \in \mathbb{R}$. If $X$ is nearly smooth, then the metric projection $P_{K_{x_{0}, c}}$ is norm-norm upper semicontinuous.

Lemma 9 (see [11]). Let $M$ be a proximal subspace. Then for any $x \in X$, one has the decomposition

$$
x=x_{1}+x_{2}, \quad x_{1} \in P_{M}(x), \quad x_{2} \in D^{-1}\left(M^{\perp}\right),
$$

where $M^{\perp}=\left\{x^{*} \in X^{*}: x^{*}(x)=0, \forall x \in M\right\}$ and

$$
D^{-1}\left(M^{\perp}\right)=\left\{x \in X: D(x) \cap M^{\perp} \neq \emptyset\right\} .
$$

If $M$ is a Chebyshev subspace, the decomposition is unique, and

$$
x=P_{M}(x)+x_{2}, \quad x_{2} \in D^{-1}\left(M^{\perp}\right) .
$$

Lemma 10. Let $X$ be a strictly convex Banach space and let $M$ be a proximal subspace. Then, for any $x \in X$, one has

$$
P_{M}(x+y)=P_{M}(x)+y, \quad y \in M
$$

Proof. Let $y \in M$, for any $z \in M$, we have that $w=z-y \in M$. Consider

$$
\begin{aligned}
& \left\|P_{M}(x)+y-(x+y)\right\| \\
& \quad=\left\|P_{M}(x)-x\right\| \leq\|w-x\| \\
& \quad=\|(w+y)-(x+y)\|=\|z-(x+y)\| .
\end{aligned}
$$

By the definition of $P_{M}$, we obtain $P_{M}(x)+y \in P_{M}(x+y)$. Since $X$ is strictly convex, we know that $P_{M}$ is single-valued, and hence we have $P_{M}(x+y)=P_{M}(x)+y$.

Similar to the proof Theorem 2.1(1) in [6], we can prove the following result by Lemmas 9 and 10 .

Lemma 11. Let $X$ be a strictly convex Banach space and let $M$ be a proximal subspace. $P$ is single-valued operator from $X$ into $M$, and $P_{M}$ is a metric projection from $X$ into $M$. Then $P=P_{M}$ if and only if the following conditions are satisfied:

(1) $P^{-1}(\theta)=D^{-1}\left(M^{\perp}\right)$;

(2) $P(x+y)=P(x)+y$, for all $y \in M$.

Theorem 12. Let $X$ be a strictly convex Banach space and let $M$ be a proximal subspace. Then the metric projection $P_{M}$ is a linear bounded operator if and only if $D^{-1}\left(M^{\perp}\right)$ is a linear subspace.

Proof. On necessity: let $P_{M}$ be a linear operator. Since $X$ is strictly convex and $M$ is proximal, then $P_{M}$ is single valued. By Lemma $11(1)$, for any $x, y \in D^{-1}\left(M^{\perp}\right)=P_{M}^{-1}(\theta), \alpha, \beta \in \mathbb{R}$, then

$$
P_{M}(\alpha x+\beta y)=\alpha P_{M}(x)+\beta P_{M}(y)=0,
$$

and hence $\alpha x+\beta y \in P_{M}^{-1}(\theta)=D^{-1}\left(M^{\perp}\right)$. This shows that $D^{-1}\left(M^{\perp}\right)$ is a linear subspace.

On sufficiency: let $D^{-1}\left(M^{\perp}\right)$ be a linear subspace and let $P_{M}$ be a metric projection; since $X$ is strictly convex, by
Lemma $11(1), P_{M}^{-1}(\theta)$ is also a linear subspace. For any $x, y \in$ $X, x-P_{M}(x), y-P_{M}(y) \in\left\{x-P_{M}(x): x \in X\right\}$, we have that

$$
\begin{aligned}
& (x+y)-\left(P_{M}(x)+P_{M}(y)\right) \\
& \quad=\left(x-P_{M}(x)\right)+\left(y-P_{M}(y)\right) \\
& \quad \in\left\{z-P_{M}(z): z \in X\right\}=P_{M}^{-1}(\theta) .
\end{aligned}
$$

By Lemma 11(2), we have that

$$
\begin{aligned}
0 & =P_{M}\left((x+y)-\left(P_{M}(x)+P_{M}(y)\right)\right) \\
& =P_{M}(x+y)-\left(P_{M}(x)+P_{M}(y)\right)
\end{aligned}
$$

It follows that $P_{M}(x+y)=P_{M}(x)+P_{M}(y)$. Note that $P_{M}$ is homogeneous; we obtain that $P_{M}$ is a linear operator. In addition, for any $x \in X$, since $\theta \in M$, we have that

$$
\begin{aligned}
\left\|P_{M}(x)\right\| & =\left\|P_{M}(x)-x+x\right\| \\
& \leq\left\|P_{M}(x)-x\right\|+\|x\| \\
& \leq\|\theta-x\|+\|x\|=2\|x\| .
\end{aligned}
$$

This shows that $P_{M}$ is a bounded operator.

\section{Conflict of Interests}

The authors declare that they have no conflict of interests.

\section{Acknowledgments}

This work was supported in part by the National Natural Science Foundation of China (Grant no. 11271248) and Scientific Research Foundation of Shanghai University of Engineering Science (Grant nos. A-0501-12-43, nhky-2012-13).

\section{References}

[1] N. V. Nevesenko, "Continuity of the diameter of a metric projection," Matematicheskie Zapiski, vol. 11, no. 4, pp. 72-82, 131-132, 1979.

[2] E. V. Oshman, "Continuity of the metric projection," Matematicheskie Zametki, vol. 37, no. 2, pp. 200-2011, 1985.

[3] J. H. Wang, "Convergence theorems for best approximations in a nonreflexive Banach space," Journal of Approximation Theory, vol. 93, no. 3, pp. 480-490, 1998.

[4] X. N. Fang and J. H. Wang, "Convexity and the continuity of metric projections," Mathematica Applicata, vol. 14, no. 1, pp. 47-51, 2001 (Chinese).

[5] Z. Zhang and Z. Shi, "Convexities and approximative compactness and continuity of metric projection in Banach spaces," Journal of Approximation Theory, vol. 161, no. 2, pp. 802-812, 2009.

[6] Y. W. Wang and J. F. Yu, "The character and representation of a class of metric projection in Banach space," Acta Mathematica Scientia A, Chinese Edition, vol. 21, no. 1, pp. 29-35, 2001.

[7] W. Song and Z. J. Cao, "The generalized decomposition theorem in Banach spaces and its applications," Journal of Approximation Theory, vol. 129, no. 2, pp. 167-181, 2004. 
[8] J. H. Wang, "The metric projections in nonreflexive Banach spaces," Acta Mathematica Scientia A, Chinese Edition, vol. 26, no. 6, pp. 840-846, 2006.

[9] R. X. Ni, "The representative of metric projection on the linear manifold in Banach spaces," Journal of Mathematical Research and Exposition, vol. 25, no. 1, pp. 99-103, 2005.

[10] J. Cabrera and B. Sadarangani, "Weak near convexity and smoothness of Banach spaces," Archiv der Mathematik, vol. 78, no. 2, pp. 126-134, 2002.

[11] Y. W. Wang and H. Wang, "Generalized orthogonal decomposition theorem in Banach space and generalized orthogonal complemented subspace," Acta Mathematica Sinica, Chinese Series, vol. 44, no. 6, pp. 1045-1050, 2001. 


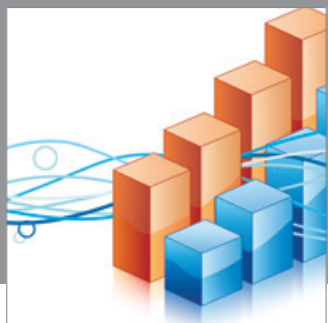

Advances in

Operations Research

mansans

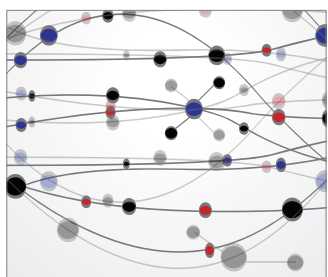

The Scientific World Journal
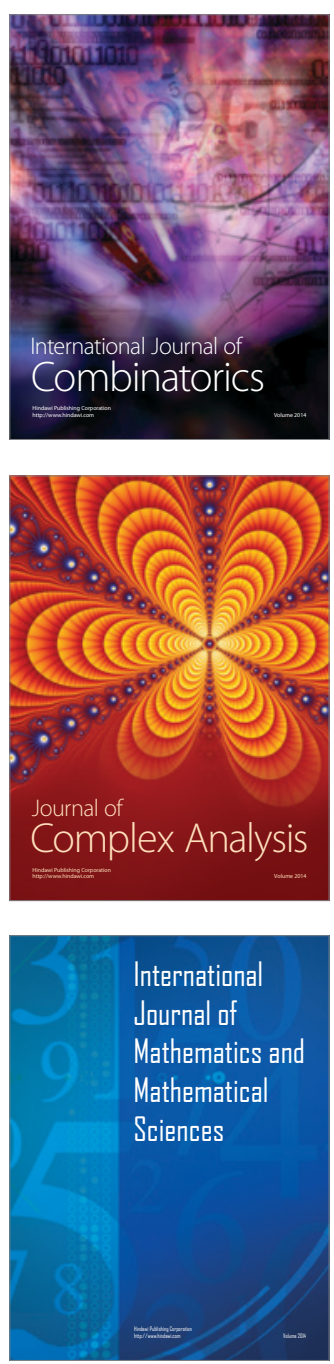
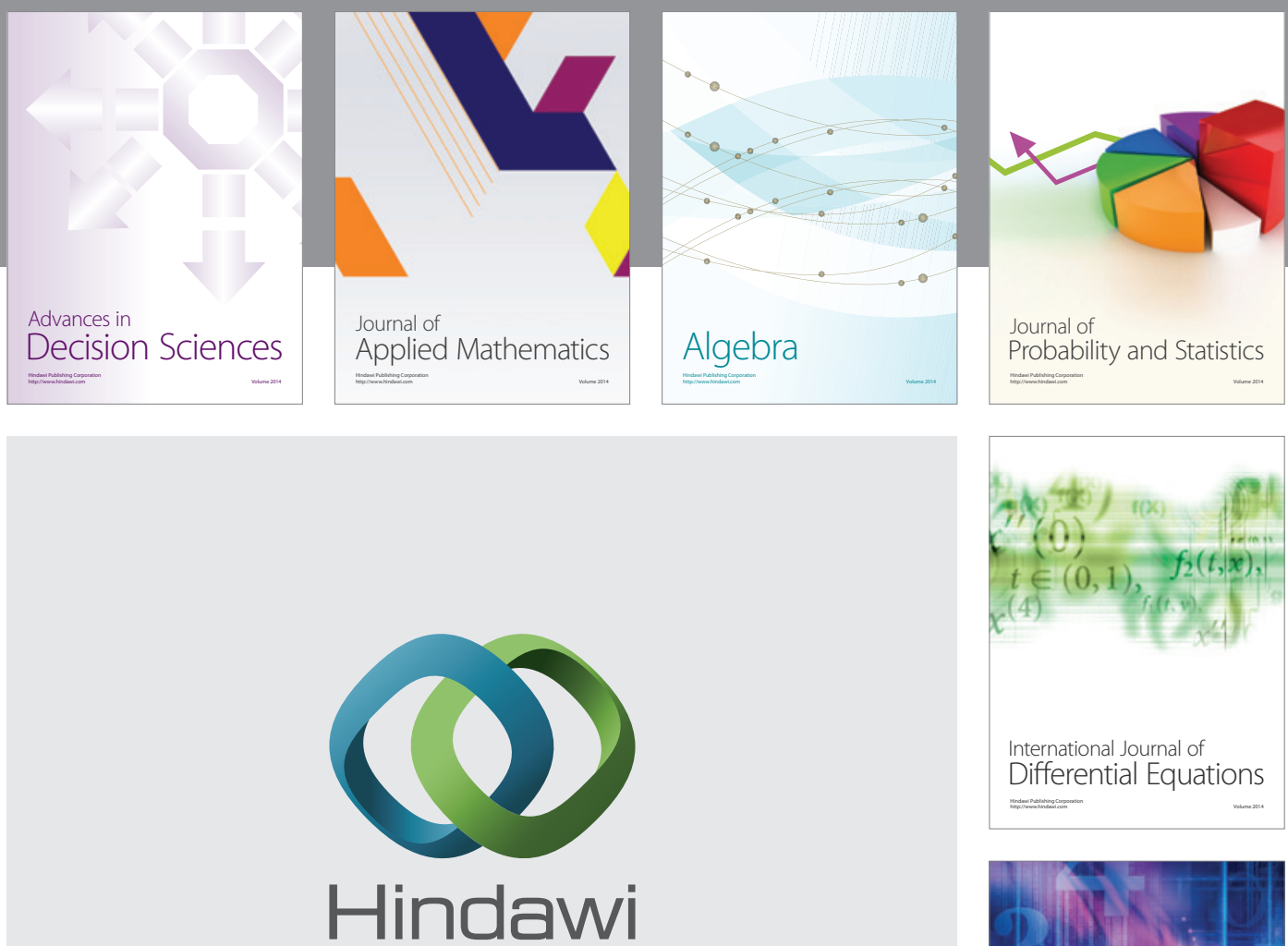

Submit your manuscripts at http://www.hindawi.com
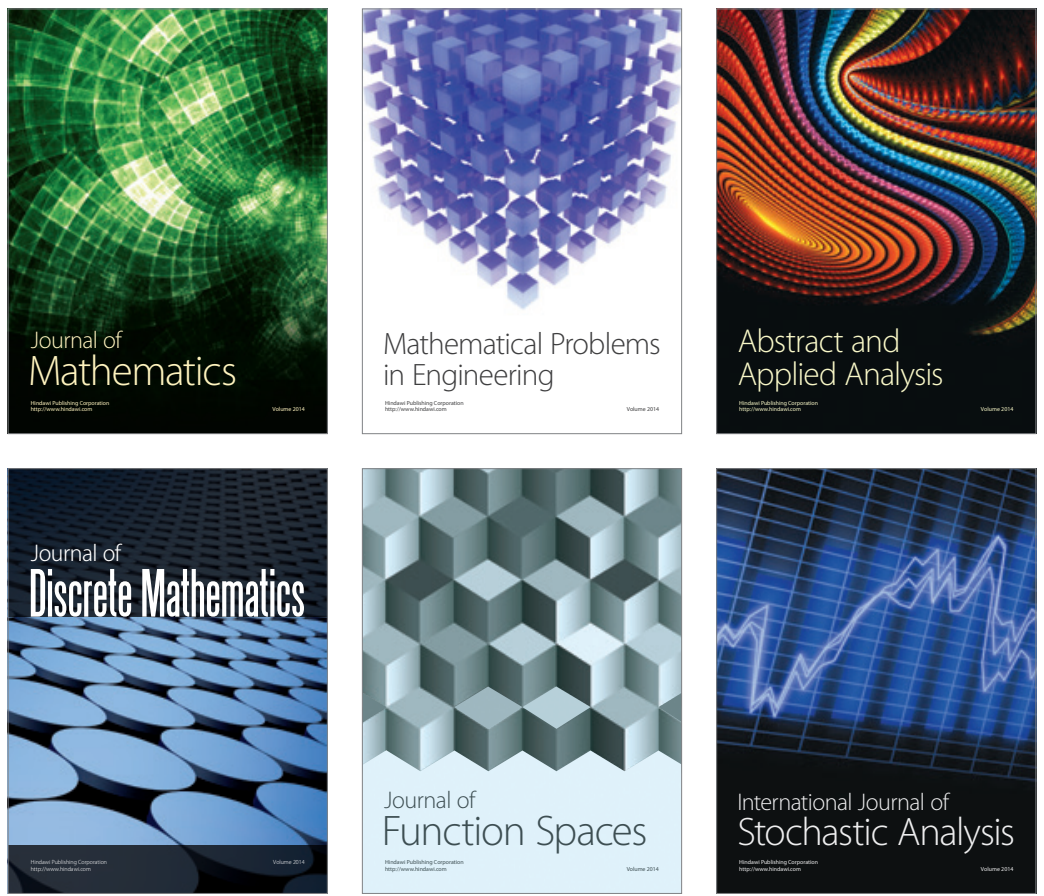

Journal of

Function Spaces

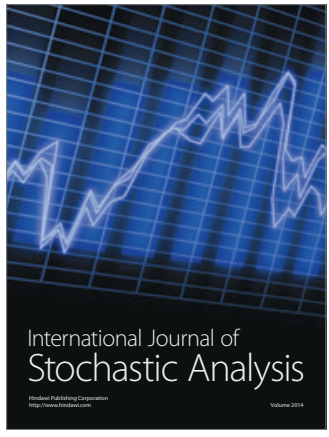

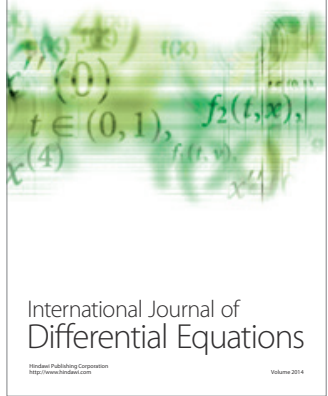
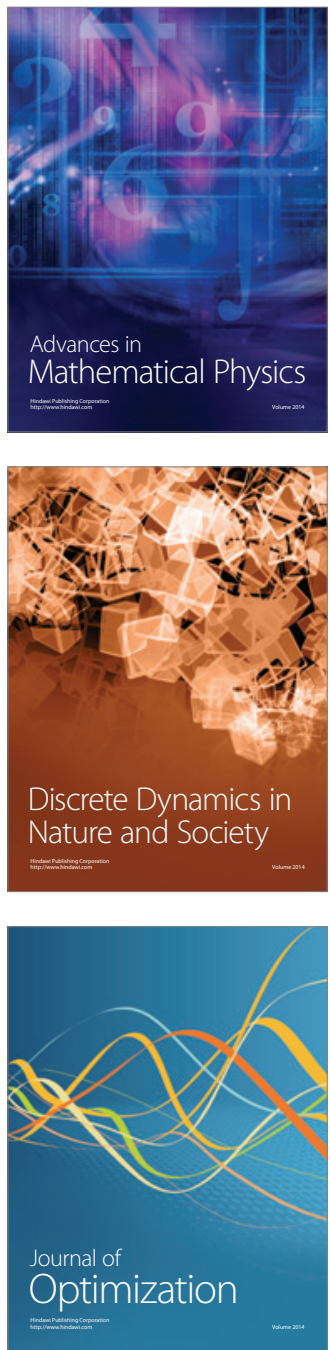